University of Wollongong

Research Online

Faculty of Engineering - Papers (Archive)

Faculty of Engineering and Information

Sciences

1998

\title{
Thermal activation and ac-field-induced discontinuous domain jumps in perovskite $\mathrm{La} 0.7 \mathrm{Ca} 0.3 \mathrm{MnO} 3$
}

Xiaolin Wang

University of Wollongong, xiaolin@uow.edu.au

J. Horvat

University of Wollongong, jhorvat@uow.edu.au

Hua-Kun Liu

University of Wollongong, hua@uow.edu.au

S. X. Dou

University of Wollongong, shi@uow.edu.au

Follow this and additional works at: https://ro.uow.edu.au/engpapers

Part of the Engineering Commons

https://ro.uow.edu.au/engpapers/246

\section{Recommended Citation}

Wang, Xiaolin; Horvat, J.; Liu, Hua-Kun; and Dou, S. X.: Thermal activation and ac-field-induced discontinuous domain jumps in perovskite La0.7Ca0.3MnO3 1998.

https://ro.uow.edu.au/engpapers/246

Research Online is the open access institutional repository for the University of Wollongong. For further information contact the UOW Library: research-pubs@uow.edu.au 


\title{
Thermal activation and ac-field-induced discontinuous domain jumps in perovskite $\mathrm{La}_{0.7} \mathrm{Ca}_{0.3} \mathrm{MnO}_{3}$
}

\author{
X. L. Wang, J. Horvat, H. K. Liu, and S. X. Dou \\ Institute for Superconducting and Electronic Materials, University of Wollongong, Wollongong, NSW 2522, Australia
}

(Received 17 November 1997)

\begin{abstract}
Low-field ac susceptibility was measured on bulk $\mathrm{La}_{0.7} \mathrm{Ca}_{0.3} \mathrm{MnO}_{3}$ prepared by a partial melting technique. Discontinuous jumps with heights of $(1-2) \times 10^{-5} \mathrm{emu}$ at temperature intervals of $3-5 \mathrm{~K}$ were observed in the ac susceptibility. 28 jumps were observed in the temperature range between $4.2 \mathrm{~K}$ and the Curie temperature of $250 \mathrm{~K}$, with an ac field amplitude of $1 \mathrm{Oe}$, a frequency of $117 \mathrm{~Hz}$, and a dc bias field of 5 and $20 \mathrm{Oe}$. Upon increasing the frequency up to $5000 \mathrm{~Hz}$, the jumps shifted to lower temperature. For a dc bias field of 50 Oe and an ac field with an amplitude of $0.1 \mathrm{Oe}$ and a frequency between 117 and $5000 \mathrm{~Hz}$, only three jumps with heights of about $3 \times 10^{-5} \mathrm{emu}$ at large temperature intervals of about $60 \mathrm{~K}$ were observed for $T<T_{c}$. With increasing frequencies, the three jumps shifted to lower temperatures without changing jump height. The discontinuous jumps in $\mathrm{La}_{0.7} \mathrm{Ca}_{0.3} \mathrm{MnO}_{3}$ were interpreted in terms of thermally and ac-field-activated domainwall jumps. [S0163-1829(98)00629-8]
\end{abstract}

Recently, the discovery of the colossal magnetoresistance (CMR) effect in perovskite-type manganese oxides $\mathrm{La}_{1-x} A_{x} \mathrm{MnO}_{3}$ (LMO) with $A=\mathrm{Ca}, \mathrm{Sr}, \mathrm{Ba}$ has attracted much interest as potential applications in magnetic recording and sensors. ${ }^{1-3}$ The Curie temperature can easily be varied by controlling doping levels and can reach $360 \mathrm{~K}$ for $\mathrm{Sr}$ doped LMO. It was observed that the occurrence of CMR at the Curie temperature with application of magnetic field is accompanied by large lattice distortion for the doped manganites, ${ }^{4}$ and magnetostriction was also observed in the CMR compounds. ${ }^{5}$ Much attention has been paid to bulk magnetization, CMR behavior, and structural properties. Since the magnetic domain behavior is a key factor for the applications, the study of domains at different external fields is very desirable. The observation of domains in La-Ca-Mn-O thin films was made by magnetic force microscopy (MFM). ${ }^{6}$ It was found that the domains were very stable at low temperatures in low dc field. We studied the ac susceptibility with various amplitudes of fields and frequencies, with a bias de field. We report discontinuous jumps in the ac susceptibility for $\mathrm{La}_{0.7} \mathrm{Ca}_{0.3} \mathrm{MnO}_{3}$ which are due to domain-wall jumps activated by thermal excitation and ac field.

High-purity powders of $\mathrm{La}_{2} \mathrm{O}_{3}, \mathrm{CaCO}_{3}$, and $\mathrm{MnCO}_{3}$ were weighed according to the nominal composition of $\mathrm{La}_{0.7} \mathrm{Ca}_{0.3} \mathrm{MnO}_{3}$. After a thorough mixing, the powders were pressed into pellets of $10 \mathrm{~mm}$ in diameter. The pellets were first sintered at temperature of $1100{ }^{\circ} \mathrm{C}$ for $24 \mathrm{~h}$, grinded into powders, pressed into pellets again, then partially melted at $1600{ }^{\circ} \mathrm{C}$ and rapidly cooled down to $900{ }^{\circ} \mathrm{C}$ with air quenching for achieving high density. After sintering in air, the samples were annealed in $\mathrm{O}_{2}$ at $600{ }^{\circ} \mathrm{C}$ for $24 \mathrm{~h}$. X-ray powder diffraction results indicated a nearly single phase for the partially melted sample. Spherical fine particles of $\mathrm{CaMnO}_{3}$ were also observed. Magnetization and ac susceptibility was measured between $4.2 \mathrm{~K}$ and room temperature using a Quantum Design physical property measurement system. ac susceptibility was obtained with an ac field of amplitude of 0.1 and 1 Oe and frequencies of 117, 500, 2000, and 5000 $\mathrm{Hz}$, in the dc bias fields of 5, 20, and $50 \mathrm{Oe}$.
Figure 1 shows the real part of magnetic susceptibility $\left(\chi^{\prime}\right)$ vs temperature for a dc field $\left(H_{\mathrm{dc}}\right)$ of $50 \mathrm{Oe}$, the amplitude of an ac field $\left(H_{\mathrm{ac}}\right)$ of $0.1 \mathrm{Oe}$ and frequencies of 117 and $2000 \mathrm{~Hz}$. It is clearly seen that a ferromagnetic transition occurs at $250 \mathrm{~K}$. In addition to this, there are two jumps in $\chi^{\prime}$ for $f=117 \mathrm{~Hz}$ (inset to Fig. 1). The higher the frequency, the lower the temperature at which these jumps appear. Another jump appeared just below the sharp transition for $f$ $=2000 \mathrm{~Hz}$. These three jumps have heights of about 3 $\times 10^{-5}$ emu with a temperature interval of $60 \mathrm{~K}$. Figure 2 shows $\chi^{\prime}$ vs temperature for $H_{\mathrm{dc}}=20 \mathrm{Oe}, H_{\mathrm{ac}}=1 \mathrm{Oe}$, and $f=117 \mathrm{~Hz}$. There is 28 jumps in $\chi^{\prime}$ with heights of $1-2$ $\times 10^{-5} \mathrm{emu}$, at temperature intervals of $3-5 \mathrm{~K}$. The jumps seem to be superimposed to the main, smooth $\chi^{\prime}$ vs $T$ curve, which can be obtained by connecting $\chi^{\prime}$ vs $T$ for $T<42 \mathrm{~K}$ with the beginning of each of the jumps (dashed line in Fig. 2). The temperature at which the jumps appear depends on the frequency in qualitatively the same way as for $H_{\mathrm{dc}}$ $=50$ Oe (inset to Fig. 2), although this dependence is about half as strong as for $H_{\mathrm{dc}}=50 \mathrm{Oe}$ (Fig. 1). Lowering $H_{\mathrm{dc}}$ to 5 Oe, the ac susceptibility vs temperature exhibited the same behavior as that for $20 \mathrm{Oe}$. The number of the jumps and temperature at which they appear do not change. However, the value of $\chi^{\prime}$ is a bit smaller than for $H_{\mathrm{dc}}=20 \mathrm{Oe}$, as shown in Fig. 3. For all these measurements, the jumps in $\chi^{\prime}$ were not accompanied by any features in the imaginary part $\left(\chi^{\prime \prime}\right)$ of ac susceptibility.

The jumps in $\chi^{\prime}$ seem to be a thermally activated phenomenon, helped by the magnetic field. While the dc field has a strong influence defining the number of the jumps (Figs. 1 and 2), the ac field excites the system in addition to thermal excitations. We suggest that the jumps in $\chi^{\prime}$ occur because of the releasing of domain walls from pinning centers under a combined influence of the dc field, thermal excitations, and the ac field. Applying a large dc field, only the strongest pinning centers in the sample are effective in pinning the domain walls, ${ }^{7-10}$ giving only two jumps in $\chi^{\prime}$ vs $T$ (Fig. 1). For smaller fields, the number of effective pinning sites is increased, giving a large number of jumps (Fig. 2). 


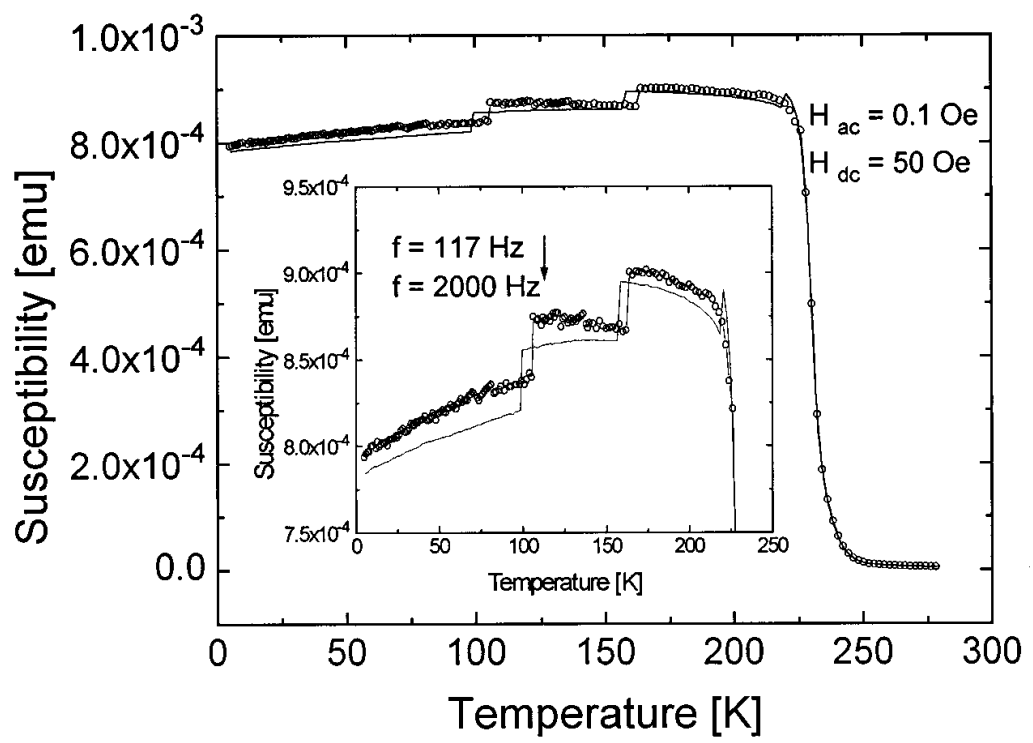

FIG. 1. Real part of magnetic susceptibility $\chi^{\prime}$ vs temperature for a dc field of 50 Oe, an amplitude of the ac field of 0.1 Oe, and frequencies of 117 and $2000 \mathrm{~Hz}$. The inset shows two jumps in $\chi^{\prime}$ shift to low temperature with increasing frequency.

The amplitude of the ac field is not large enough to release pinned domain walls. It merely shakes within the potential well.

The energy of thermal excitation is proportional to temperature $(T)$ :

$$
E_{t}=k T,
$$

whereas the energy of the excitation by the harmonic ac field is proportional to the square of its frequency $(f)$ :

$$
E_{\mathrm{ac}}=c f^{2} \text {. }
$$

Domain walls get released from pinning centers when the pinning energy $\left(E_{p}\right)$ is equal to the combined energy of $\mathrm{dc}$ field $\left(E_{\mathrm{dc}}\right)$, thermal, and ac field excitations:

$$
E_{p}=E_{\mathrm{dc}}+E_{t}+E_{\mathrm{ac}} .
$$

Combining Eqs. (1)-(3), one obtains

$$
k T+c f^{2}=E_{p}-E_{\mathrm{dc}} .
$$

For a constant dc field, Eq. (4) gives the temperature at which the domain walls get released from pinning centers:

$$
T_{0}=\alpha-\beta f^{2},
$$

where $\alpha=\left(E_{p}-E_{\mathrm{dc}}\right) / k$ and $\beta=c / k$.

Scanning electron microscopy (SEM) and energy dispersive analysis (EDA) observations of our samples revealed spherical fine particles of $\mathrm{CaMnO}_{3}$ (about $1 \mu \mathrm{m}$ in diameter).

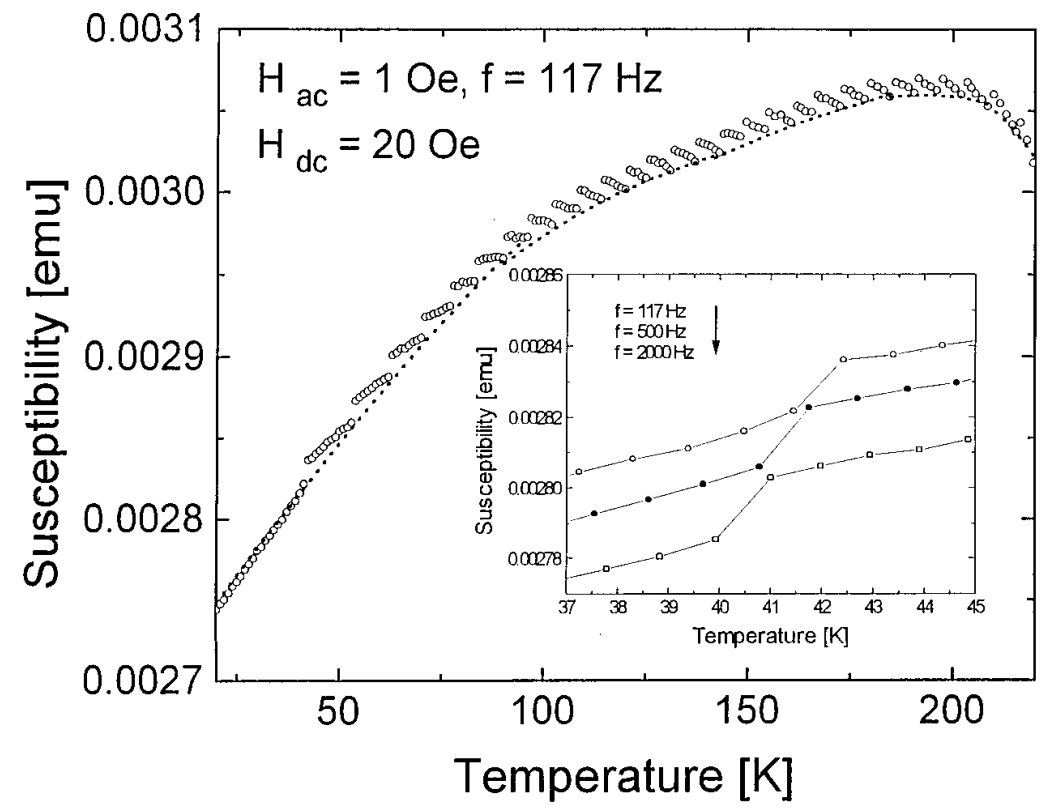

FIG. 2. $\chi^{\prime}$ vs temperature for $H_{\mathrm{dc}}=20 \mathrm{Oe}, H_{\mathrm{ac}}=1 \mathrm{Oe}$, and $f=117 \mathrm{~Hz}$. The inset shows that the temperature at which the jumps appear depends on the frequency in qualitatively the same way as for $H_{\mathrm{dc}}=50 \mathrm{Oe}$ (inset to Fig. 2), 




FIG. 3. $\chi^{\prime}$ vs temperature for $H_{\mathrm{dc}}=5$ Oe (open circle) and $H_{\mathrm{dc}}=20$ Oe (line) with $H_{\mathrm{ac}}=1 \mathrm{Oe}$ and $f=117 \mathrm{~Hz}$.

These particles are probably responsible for the domain-wall pinning and the observed jumps in $\chi^{\prime}$. If there was no pinning centers in the sample, the domain walls would under the influence of external field take an equilibrium position with minimum energy. With pinning centers, domain walls are pinned in different positions and the field exerts a pressure on them tending to shift them into equilibrium position. The further away they are from the equilibrium position, the larger the pressure is. However, pinned domain walls can still move within the potential well of pinning centers. The smaller the pressure by the field, the larger the mobility of domain walls in the potential well.

When a domain wall gets released from a pinning site under the combined influence of the dc field, thermal excitations, and the ac field, it will jump to a new stable position. ${ }^{7-9}$ In the new position, it will experience a smaller pressure due to the dc field than in the old position, ${ }^{7}$ resulting in an increased mobility of the domain wall in the new position. Since the increased mobility gives larger in-phase ac susceptibility, jumps in $\chi^{\prime}$ will occur at a temperature at

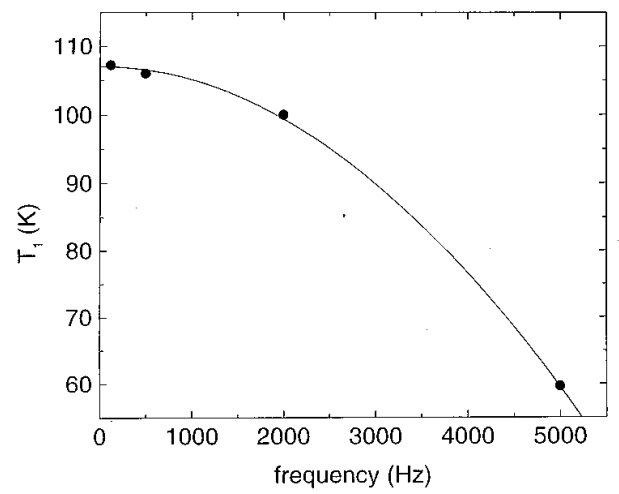

FIG. 4. Plot of the temperature of the first jump in Fig. 1 as a function of frequency. The solid line is a fit using Eq. (5). which the domain walls jump to new pinning sites. Jumping back to old sites will not occur because the pressure on the domain walls due to the dc field pushes them in one direction only, unlike the dc and thermal excitations. We stress that the measured jumps in $\chi^{\prime}$ will not be accompanied by jumps in $\chi^{\prime \prime}$. This is because after the jump, the domain walls only oscillate in new potential wells, which is a reversible process. They do not have enough energy to jump continuously between the pinning sites. The energy loss due to the single jump from old to new pinning sites is not measured in our experiment because the susceptibility is measured at fixed temperature intervals, averaged over several seconds, while the jumps occur in a much shorter time. A peak in $\chi^{\prime \prime}$ would be obtained, however, if the measurement was performed in that short time, during the jump. Our measuring system cannot detect such fast events. The number of jumps in our experiment is not an intrinsic property of the material. It depends on the distribution and strength of the pinning centers in the sample.

If our model is correct, the jumps in $\chi^{\prime}$ vs temperature should occur at $T_{0}$ in Eq. (5). To check this, we plot the temperature of the first jump in Fig. 1 as a function of frequency (Fig. 4). The solid line is a fit using Eq. (5). Apparently, our data show very good agreement with Eq. (5), giving $\alpha=107 \mathrm{~K}$ and $\beta=1.9 \times 10^{-6} \mathrm{~K} / \mathrm{Hz}^{2}$. The jump at higher temperature in Fig. 1 gives $\alpha=164 \mathrm{~K}$ and $\beta=1.48$ $\times 10^{-6} \mathrm{~K} / \mathrm{Hz}^{2}$.

In conclusion, we showed that the observed jumps in $\chi^{\prime}$ vs $T$ occur because of the thermal excitation of the system, combined with the harmonic excitation of the ac field. We suggested that they appear when domain walls get released from pinning centers under these excitations, resulting in a sudden increase of their mobility.

X.L.W. thanks the Department of Education, Employment and Youth Affairs of Australia and the University of Wollongong for financial Support with OPRS and UPA. 
${ }^{1}$ S. Jin, T. H. Hiefel, M. McCormack, R. A. Fastnacht, R. Ramesh, and L. H. Chen, Science 264, 413 (1994).

${ }^{2}$ R. von Helmholt, J. Wecker, B. Holszapfel, L. Schultz, and K. Samwer, Phys. Rev. Lett. 71, 2331 (1993).

${ }^{3}$ M. A. Subramanian, B. H. Toby, A. P. Ramirez, W. J. Marshall, A. W. Sleight, and G. H. Kwei, Science 273, 81 (1996).

${ }^{4}$ J. M. De Teresa, M. R. Ibarra, P. A. Algarabel, C. Ritter, C. Marquina, J. Blasco, J. Garcia, A. del Moral, and Z. Arnold, Nature (London) 386, 256 (1997).

${ }^{5}$ J. M. De Teresa, J. Blasco, M. R. Ibarra, J. Garcia, C. Marquina, P. Algarabel, and A. del Moral, J. Appl. Phys. 79, 5175 (1996).
${ }^{6}$ Qingyou Lu, Chun-Che Chen, and Alex de Lozanne, Science 276, 2006 (1997).

${ }^{7}$ S. Chikazumi, The Physics of Ferromagnetism. Magnetic Properties and Practical Applications (Mir, Moscow, 1986), p. 248.

${ }^{8}$ J. Horvat, E. Babic, Z. Marohnic, and H. H. Liebermann, J. Magn. Magn. Mater. 87, 339 (1990).

${ }^{9}$ D. C. Jiles and D. L. Atherton, J. Magn. Magn. Mater. 61, 48 (1986).

${ }^{10}$ J. Horvat, E. Babic, and Z. Marohnic, J. Magn. Magn. Mater. 86, L1 (1990). 